\title{
Quantum Algorithms for Quantum Field Theories
}

\author{
Stephen P. Jordan, ${ }^{1}$ Keith S. M. Lee, ${ }^{2}$ John Preskill ${ }^{3}$ \\ ${ }^{1}$ National Institute of Standards and Technology, Gaithersburg, MD \\ ${ }^{2}$ University of Pittsburgh, Pittsburgh, PA \\ ${ }^{3}$ California Institute of Technology, Pasadena, CA
}

\begin{abstract}
Quantum field theory reconciles quantum mechanics and special relativity, and plays a central role in many areas of physics. We develop a quantum algorithm to compute relativistic scattering probabilities in a massive quantum field theory with quartic self-interactions ( $\phi^{4}$ theory) in spacetime of four and fewer dimensions. Its run time is polynomial in the number of particles, their energy, and the desired precision, and applies at both weak and strong coupling. In the strong-coupling and high-precision regimes, our quantum algorithm achieves exponential speedup over the fastest known classical algorithm.
\end{abstract}

The question whether quantum field theories can be efficiently simulated by quantum computers was first posed by Feynman three decades ago when he introduced the notion of quantum computers (1). Since then, efficient quantum algorithms have been developed to simulate the dynamics of quantum lattice models and quantum systems with a fixed number of particles (2-7), but the question about quantum field theories has remained open.

In this paper, we show that quantum computers can efficiently calculate scattering probabilities in continuum $\phi^{4}$ theory to an arbitrary degree of precision. We choose $\phi^{4}$ theory because it 
is among the simplest interacting quantum field theories, and thus illustrates the essential issues without unnecessary complications. We simulate a process in which initially well-separated particles with well-defined momentum scatter off each other.

In our algorithm, we introduce several new techniques. First, we show that the field can be accurately represented with finitely many qubits by discretization of space via a lattice, and discretization of the field value at each lattice site. Analyzing spatial discretization errors is highly non-trivial for quantum field theories because of renormalization. We approach this problem using effective field theory. Secondly, we must create the initial state for the simulation. We do so by developing a modified version of adiabatic state preparation suitable for preparing non-eigenstates, such as wavepackets. Thirdly, to improve the efficiency of simulating the time evolution, we show that Suzuki-Trotter formulae converge faster in cases where the underlying Hamiltonians have spatial locality. These techniques may be of independent interest, beyond their application to simulating quantum field theory.

No previous paper has addressed the quantum computation of scattering amplitudes or the convergence of quantum simulations to the continuum limit of a quantum field theory. The issue of gauge symmetries in qubit representations of lattice field theories has been studied (8), and there is an extensive literature on how experimentally to construct Hamiltonians that approximate lattice gauge theories, in systems of atoms or superconducting qubits (see, for example, (9-19)). These previous studies are on the experimental analog implementation of lattice Hamiltonians, whereas the present work addresses digital simulation, with explicit consideration of convergence to the continuum, and efficient preparation of wavepacket states for the computation of dynamical quantities such as scattering probabilities.

The input to our algorithm is a list of the momenta of the incoming particles, and the output is a list of the momenta of the outgoing particles produced by the physical scattering process. At relativistic energies, the number of outgoing particles may differ from the number of incoming 
particles. However, because we consider only the case of non-zero particle mass, the number of outgoing particles is at most linear in the center-of-mass energy of the incoming particles. In accordance with quantum mechanics, the incoming momenta do not uniquely determine the outgoing momenta, but rather a probability distribution over possible outcomes. Upon repeated runs, our quantum algorithm samples from this distribution. We quantify the precision of our simulation by demanding that the probability of a given outcome from the simulation differ from the true physical probability by no more than $\pm \epsilon$.

The scattering processes simulated closely match experiments in particle accelerators, which are the standard tools to probe uniquely quantum field-theoretical effects. The problem of calculating the scattering amplitudes, encoded in an object called the $S$-matrix, has consequently been well studied.

In complexity theory, the efficiency of an algorithm is judged by how its computational demands scale with the problem size or some other quantity associated with the problem's intrinsic difficulty. An algorithm with polynomial-time asymptotic scaling is considered to be feasible, whereas one with super-polynomial (typically, exponential) scaling is considered infeasible. This classification has proved to be a very useful guide in practice. Our results can be roughly summarized as follows: the calculation of quantum field-theoretical scattering amplitudes at high precision or strong coupling is infeasible on classical computers with known techniques but feasible on quantum computers.

Traditional calculations of QFT scattering amplitudes rely upon perturbation theory, namely, a series expansion in powers of the coupling (the coefficient of the interaction term), which is taken to be small. A powerful and intuitive way of organizing this perturbative expansion is through Feynman diagrams, in which the number of loops is associated with the power of the coupling. A reasonable measure of the computational complexity of perturbative calculations is therefore the number of Feynman diagrams, which is determined by combinatorics, and grows 
factorially with the number of loops and the number of external particles.

If the coupling constant is insufficiently small, the perturbation series does not yield correct results. In $\phi^{4}$ theory, for $D=2,3$ spacetime dimensions, by increasing the coupling $\lambda_{0}$, one eventually reaches a quantum phase transition at some critical coupling $\lambda_{c}(20-22)$. In the parameter space near this phase transition, perturbative methods become unreliable; this region is referred to as the strong-coupling regime. There are then no known feasible classical methods for calculating scattering amplitudes, although lattice field theory can be used to obtain static quantities, such as mass ratios. Even at weak coupling, the perturbation series is not convergent, although it is asymptotic (23-25). Including higher-order contributions beyond a certain point makes the approximation worse. There is thus a maximum possible precision achievable perturbatively.

We find that the number of quantum gates, $G_{\text {weak }}$, needed to sample from scattering probabilities in weakly coupled, $(d+1)$-dimensional $\phi^{4}$ theory with accuracy $\pm \epsilon$ scales as follows ${ }^{1}$ :

$$
G_{\text {weak }} \sim \begin{cases}\left(\frac{1}{\epsilon}\right)^{1.5+o(1)}, & d=1, \\ \left(\frac{1}{\epsilon}\right)^{2.376+o(1)}, & d=2, \\ \left(\frac{1}{\epsilon}\right)^{3.564+o(1)}, & d=3 .\end{cases}
$$

The asymptotic scaling of the number of gates used to simulate the strongly coupled theory is summarized in Table 1.

Although quantum field theory is typically expressed in terms of Lagrangians, and within the interaction picture, our algorithm is more naturally described in the formalism of Hamiltonians, and within the Schrödinger picture. We start by defining a lattice $\phi^{4}$ theory, and subsequently address convergence to the continuum theory. (In $D=4$, the continuum limit is believed to be the free theory. Nevertheless, since the coupling shrinks only logarithmically, scattering processes for particles with small momenta in lattice units are still interesting to compute.)

\footnotetext{
${ }^{1} f(n)=o(g(n))$ if and only if $\lim _{n \rightarrow \infty} f(n) / g(n)=0$. In the case of $\epsilon$ scaling it is of course $1 / \epsilon$ that is taken to infinity. We have used little-o notation to simplify our exposition. For more technical detail, see Appendix A.
} 


\begin{tabular}{|c|c|c|c|}
\hline & $\lambda_{c}-\lambda_{0}$ & $p$ & $n_{\text {out }}$ \\
\hline$d=1$ & $\left(\frac{1}{\lambda_{c}-\lambda_{0}}\right)^{8+o(1)}$ & $p^{4+o(1)}$ & $\tilde{O}\left(n_{\text {out }}^{5}\right)$ \\
\hline$d=2$ & $\left(\frac{1}{\lambda_{c}-\lambda_{0}}\right)^{5.04+o(1)}$ & $p^{6+o(1)}$ & $\tilde{O}\left(n_{\text {out }}^{7.128}\right)$ \\
\hline
\end{tabular}

Table 1: The asymptotic scaling of the number of quantum gates needed to simulate scattering in the strong-coupling regime in one and two spatial dimensions is polynomial in $p$, the momentum of the incoming pair of particles, $\lambda_{c}-\lambda_{0}$, the distance from the phase transition, and $n_{\text {out }}$, the maximum kinematically allowed number of outgoing particles. The notation $f(n)=\tilde{O}(g(n))$ means $f(n)=O\left(g(n) \log ^{c}(n)\right)$ for some constant $c$.

Let $\Omega=a \mathbb{Z}_{\hat{L}}^{d}$, that is, an $\hat{L} \times \ldots \times \hat{L}$ lattice in $d$ spatial dimensions with periodic boundary conditions and lattice spacing $a$. The number of lattice sites is $\mathcal{V}=\hat{L}^{d}$. For each $\mathrm{x} \in \Omega$, let $\phi(\mathbf{x})$ be a continuous, real degree of freedom - interpreted as the field at $\mathbf{x}-$ and $\pi(\mathbf{x})$ the corresponding canonically conjugate variable. In canonical quantization, these degrees of freedom are promoted to Hermitian operators with the commutation relation

$$
[\phi(\mathbf{x}), \pi(\mathbf{y})]=i a^{-d} \delta_{\mathbf{x}, \mathbf{y}} \mathbb{1}
$$

As is standard in quantum field theory, we use units with $\hbar=c=1$. $\phi^{4}$ theory on the lattice $\Omega$ is defined by the Hamiltonian

$$
H=\sum_{\mathbf{x} \in \Omega} a^{d}\left[\frac{1}{2} \pi(\mathbf{x})^{2}+\frac{1}{2}\left(\nabla_{a} \phi\right)^{2}(\mathbf{x})+\frac{1}{2} m_{0}^{2} \phi(\mathbf{x})^{2}+\frac{\lambda_{0}}{4 !} \phi(\mathbf{x})^{4}\right],
$$

where $\nabla_{a} \phi$ denotes a discretized derivative, that is, a finite-difference operator.

We represent the state of the lattice field theory by devoting one register of qubits to store the value of the field at each lattice point. Each $\phi(\mathbf{x})$ is in principle an unbounded continuous variable. To represent the field at a given site with finitely many qubits, we cut off the field at a maximum magnitude $\phi_{\max }$ and discretize it in increments of $\delta_{\phi}$. This requires $n_{b}=O\left(\log \left(\phi_{\max } / \delta_{\phi}\right)\right)$ qubits per site. Note that this field discretization is a separate issue from the spatial discretization via the lattice $\Omega$. 
Let $|\psi\rangle$ be any state such that $\langle\psi|H| \psi\rangle \leq E$. The probability distribution over $\phi(\mathbf{x})$ defined by $|\psi\rangle$ (for any $\mathbf{x} \in \Omega$ ) has a very low probability ${ }^{2}$ for $|\phi(\mathbf{x})|$ to be much larger than $O(\sqrt{E})$. Thus, a cutoff $\phi_{\max }=O\left(\sqrt{\frac{\mathcal{V} E}{a^{d} m_{0}^{2} \epsilon}}\right)$ suffices to ensure fidelity $1-\epsilon$ to the original state $|\psi\rangle$. One can prove this by bounding $\langle\psi|\phi(\mathbf{x})| \psi\rangle$ and $\left\langle\psi\left|\phi^{2}(\mathbf{x})\right| \psi\right\rangle$ as functions of $E$ and applying Chebyshev's inequality (§A.4). To choose $\delta_{\phi}$, note that the eigenbasis of $a^{d} \pi(\mathbf{x})$ is the Fourier transform of the eigenbasis of $\phi(\mathbf{x})$. Hence, discretizing $\phi(\mathbf{x})$ in units of $\delta_{\phi}$ is equivalent to introducing the cutoff $-\pi_{\max } \leq \pi(\mathbf{x}) \leq \pi_{\max }$, where $\pi_{\max }=\frac{1}{a^{d} \delta_{\phi}}$. By bounding the expectations of $\pi(\mathbf{x})$ and $\pi^{2}(\mathbf{x})$, one finds that it suffices to choose $\pi_{\max }=O\left(\sqrt{\frac{\mathcal{V E}}{\epsilon a^{d}}}\right)$, and thus $n_{b}=O\left(\log \left(\frac{\mathcal{V} E}{m_{0} \epsilon}\right)\right)$.

We now turn to the main three tasks of quantum simulation: preparing an initial state, simulating the time evolution $e^{-i H t}$, and measuring final observables. We discuss simulation of time evolution first, as it is used in all three tasks. The unitary operator $e^{-i H t}$ can be approximated by a quantum circuit of $O\left((t \mathcal{V})^{1+1 / 2 k}\right)$ gates implementing a $k^{\text {th }}$-order Suzuki-Trotter formula of the type described in $(4,26)$. This near-linear scaling with $t$ has long been known. The scaling with $\mathcal{V}$ is a consequence of the locality ${ }^{3}$ of $H$ (§A.6) and appears not to have been noted previously in the quantum algorithms literature.

To simulate scattering, one needs to prepare an initial state of particles in well-separated wavepackets. We do so by preparing the vacuum of the $\lambda_{0}=0$ theory, exciting wavepackets, and then adiabatically turning on the coupling $\lambda_{0}$. Let $H^{(0)}$ be the Hamiltonian obtained by setting $\lambda_{0}=0$ in $H . H^{(0)}$ defines an exactly solvable model in which the particles are noninteracting. The vacuum (ground) state $|\operatorname{vac}(0)\rangle$ of $H^{(0)}$ is a multivariate Gaussian wavefunction in the variables $\{\phi(\mathbf{x}) \mid \mathbf{x} \in \Omega\}$, and can therefore be prepared using the method of Kitaev and Webb (27). The asymptotic scaling of the Kitaev-Webb method is dictated by the computa-

\footnotetext{
${ }^{2}$ For $\lambda_{0}>0$ one has a tighter bound. In this case it is unlikely for $|\phi(\mathbf{x})|$ to be much larger than $O\left(E^{1 / 4}\right)$ (§A.4).

${ }^{3} H$ couples only nearest-neighbor sites, via the $\left(\nabla_{a} \phi\right)^{2}$ term.
} 
tion of the $\mathbf{L D L}^{T}$ decomposition of the covariance matrix, which can be done classically in $O\left(\mathcal{V}^{2.376}\right)$ time with $(28,29)$.

In analogy with the familiar case of the harmonic oscillator, one can define creation and annihilation operators $a_{\mathbf{p}}$ and $a_{\mathbf{p}}^{\dagger}$ such that $H^{(0)}=\sum_{\mathbf{p} \in \Gamma} L^{-d} \omega_{\mathbf{p}} a_{\mathbf{p}}^{\dagger} a_{\mathbf{p}}+E^{(0)} \mathbb{1}$, where $\Gamma=\frac{2 \pi}{\hat{L} a} \mathbb{Z}_{\hat{L}}^{d}$ is the momentum-space lattice corresponding to $\Omega, \omega_{\mathbf{p}}=\sqrt{m_{0}^{2}+\frac{4}{a^{2}} \sum_{j=1}^{d} \sin ^{2}\left(\frac{a p_{j}}{2}\right)}$, and $E^{(0)}$ is an irrelevant zero-point energy. The operator $a_{\mathbf{p}}^{\dagger}$ can be interpreted as creating a (completely delocalized) particle of the non-interacting theory with momentum $\mathbf{p}$ and energy $\omega_{\mathbf{p}}$.

The (unnormalized) state $\phi(\mathbf{x})|\operatorname{vac}(0)\rangle$ is interpreted as a single particle localized at $\mathbf{x}$ (see, e.g., (30)). Because $a_{\mathbf{p}}|\operatorname{vac}(0)\rangle=0, \phi(\mathbf{x})|\operatorname{vac}(0)\rangle=a_{\mathbf{x}}^{\dagger}|\operatorname{vac}(0)\rangle$, where

$$
a_{\mathbf{x}}^{\dagger}=\sum_{\mathbf{p} \in \Gamma} L^{-d} e^{-i \mathbf{p} \cdot \mathbf{x}} \sqrt{\frac{1}{2 \omega(\mathbf{p})}} a_{\mathbf{p}}^{\dagger} .
$$

The operator

$$
a_{\psi}^{\dagger}=\eta(\psi) \sum_{\mathbf{x} \in \Omega} a^{d} \psi(\mathbf{x}) a_{\mathbf{x}}^{\dagger}
$$

creates a wavepacket with position-space wavefunction $\psi \cdot(\eta(\psi)$ is a normalization constant, chosen so that $\left[a_{\psi}, a_{\psi}^{\dagger}\right]=1$.) $a_{\psi}^{\dagger}$ is not unitary, so it cannot be directly implemented by a quantum circuit. Instead, we introduce an ancillary qubit and let

$$
H_{\psi}=a_{\psi}^{\dagger} \otimes|1\rangle\left\langle 0\left|+a_{\psi} \otimes\right| 0\right\rangle\langle 1|
$$

One can verify that $e^{-i H_{\psi} \pi / 2}|\operatorname{vac}(0)\rangle|0\rangle=-i a_{\psi}^{\dagger}|\operatorname{vac}(0)\rangle|1\rangle$. Using a high-order Suzuki-Trotter formula $(4,26)$, we can construct an efficient quantum circuit approximating the unitary transformation $e^{-i H_{\psi} \pi / 2}$. Applied to $|\operatorname{vac}(0)\rangle$, this circuit yields the desired state up to an irrelevant global phase and an unentangled ancillary qubit, which can be discarded. We repeat this process for each incoming particle desired.

Because we wish to create localized wavepackets, we can choose $\psi(\mathbf{x})$ to have bounded support. Expanding $a_{\psi}^{\dagger}$ in terms of the operators $\phi$ and $\pi$ yields an expression of the form 
$a_{\psi}^{\dagger}=\sum_{\mathbf{x} \in \Omega}[f(\mathbf{x}) \phi(\mathbf{x})+g(\mathbf{x}) \pi(\mathbf{x})]$, where $f(\mathbf{x})$ and $g(\mathbf{x})$ are exponentially decaying with characteristic length scale $1 / m_{0}$ outside the support of $\psi$. Thus, $a_{\psi}$ and $a_{\psi}^{\dagger}$ can be exponentially well approximated by linear combinations of the operators $\phi$ and $\pi$ on a local region of space, and the complexity of simulating $e^{-i H_{\psi} \pi / 2}$ does not scale with the volume $V$. Furthermore, provided the initial wavepackets are separated by a distance that is large compared with $1 / m_{0}$, the preparation of each additional wavepacket leaves the existing wavepackets almost perfectly undisturbed.

At this point, we have finished constructing wavepackets of the non-interacting theory. We next use a Suzuki-Trotter formula to construct a quantum circuit simulating the unitary transformation induced by a time-dependent Hamiltonian in which the coupling constant is gradually increased from zero to its final value, $\lambda_{0}$. By the adiabatic theorem, sufficiently slow turn-on ensures that no stray particles are created during this process, provided particle creation costs energy, that is, the particles have non-zero mass. In the free theory, the particle mass is $m_{0}$. In the interacting theory, with fixed $m_{0}$ and sufficiently large $\lambda_{0}$, the mass vanishes. This marks the location of the $\phi \rightarrow-\phi$ symmetry-breaking transition. In this paper we restrict our attention to simulations within the symmetric phase, although we do consider systems arbitrarily close to the phase transition, as these should be particularly hard to simulate classically.

As Eq. 5 shows, wavepackets are not eigenstates of $H^{(0)}$. During the adiabatic turn-on, the different eigenstates acquire different dynamical phases. Thus, as the wavepacket time evolves, it propagates and broadens. This behavior is undesirable in our simulation, because we do not wish the particles to collide and scatter before the coupling reaches its final value. We therefore introduce backward time evolutions governed by time-independent Hamiltonians into the adiabatic state-preparation process to undo the dynamical phases. Specifically, let $H(s)$ parameterize the adiabatic time evolution, with $H(0)=H^{(0)}$ and $H(1)=H$. We divide the adiabatic preparation into $J$ steps, with $U_{j}$ denoting the unitary time evolution induced by the 
time-dependent Hamiltonian linearly interpolating between $H((j-1) / J)$ and $H(j / J)$ over a period of $\tau / J$. Let $M_{j}$ consist of backward, forward, and backward evolutions, namely,

$$
M_{j}=\exp \left[i H\left(\frac{j+1}{J}\right) \frac{\tau}{2 J}\right] U_{j} \exp \left[i H\left(\frac{j}{J}\right) \frac{\tau}{2 J}\right] .
$$

Our full state-preparation process is $\prod_{j=1}^{J} M_{j}$. The dynamical phases converge to zero as $J \rightarrow$ $\infty$, while the adiabatic change of eigenbasis is undisturbed ( $(\mathrm{A} .5)$.

After the system has evolved for a period in which scattering occurs, measurement is performed as follows. The interaction is adiabatically turned off, through the time-reversed version of the turn-on described above. Once we return to the free theory, we can measure the number operators of the momentum modes, using the method of phase estimation, that is, by simulating $e^{i L^{-d} a_{\mathbf{p}}^{\dagger} a_{\mathbf{p}} t}$ for various values of $t$ and Fourier transforming the results (31).

Having described how, once discretized, a quantum field theory becomes essentially an ordinary many-body quantum-mechanical system, whose evolution can be efficiently simulated on quantum computers by combining established primitives, we now consider discretization errors. To analyse the errors introduced to our simulation by discretization, we use methods of effective field theory, a well-developed formalism underlying our modern understanding of quantum field theory.

In its regime of validity, typically below a particular energy scale, an effective field theory (EFT) reproduces the behavior of the full (that is, fundamental) theory under consideration: it can be regarded as the low-energy limit of that theory. An EFT for a full theory is thus somewhat analogous to a Taylor series for a function. It involves an expansion in some suitable small parameter, so that, although it consists of infinitely many terms, higher-order terms are increasingly suppressed. Thus, the series can be truncated, with corresponding finite and controllable errors.

We apply this framework to analyse the effect of discretizing the spatial dimensions of the 
continuum $\phi^{4}$ quantum field theory. The discretized Lagrangian can be thought of as the leading contribution (denoted by $\mathcal{L}^{(0)}$ ) to an effective field theory. From the leading operators left out we can thus infer the scaling of the error associated with a non-zero lattice spacing, $a$.

The full (untruncated) effective Lagrangian will have every coupling respecting the $\phi \rightarrow-\phi$ symmetry, and so will take the form

$$
\mathcal{L}_{\text {eff }}=\mathcal{L}^{(0)}+\frac{c}{6 !} \phi^{6}+c^{\prime} \phi^{3} \partial^{2} \phi+\frac{c^{\prime \prime}}{8 !} \phi^{8}+\cdots .
$$

This can be simplified. First, the chain rule and integration by parts (with boundary terms dropped) can be used to write any operator with two derivatives acting on different fields in the form $\phi^{n} \partial^{2} \phi$. For example, $\phi^{2} \partial_{\mu} \phi \partial^{\mu} \phi=\frac{1}{3} \partial_{\mu}\left(\phi^{3}\right) \partial^{\mu} \phi \rightarrow-\frac{1}{3} \phi^{3} \partial^{2} \phi$. Such an operator can then be simplified via the equation of motion $(32,33)$. If this were the equation of motion of the continuum theory, any derivative operator would then be completely eliminated. In the discretized theory, however, the equation of motion is modified and there are residual, Lorentzviolating operators. In fact, because the difference operators in the discretized theory are only approximately equal to the derivatives in the continuum theory, the simplest Lorentz-violating operators are induced purely by discretization.

In units where $\hbar=c=1$, all quantities have units of some power of mass. The mass dimensions (denoted by [.]) of the field and coupling in $D=d+1$ spacetime dimensions are $[\phi]=\frac{D-2}{2}$ and $[\lambda]=4-D$, which imply that

$$
[c]=6-2 D, \quad\left[c^{\prime \prime}\right]=8-3 D .
$$

In $D=4$ dimensions, $[c]=-2$ and $\left[c^{\prime \prime}\right]=-4$. Since the only relevant dimensionful parameter is the lattice spacing, that is, $\Lambda \sim \pi / a$, this means that $c \sim a^{2}$ and $c^{\prime \prime} \sim a^{4}$. We see then that, of the operators not included in the Lagrangian $\mathcal{L}^{(0)}, \phi^{6}$ is more significant than $\phi^{2 n}$, for $n>3$.

In $D=2,3$, the scaling of the coefficients with $a$ is somewhat less obvious, because now the coupling $\lambda$ provides another dimensionful parameter. To obtain the scaling of $c$, one should 
consider the Feynman diagram that generates the corresponding operator. This involves three $\phi^{4}$ vertices, so

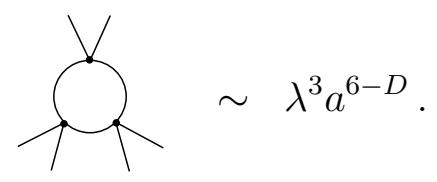

(Other diagrams involve higher powers of $\lambda$ and hence are suppressed by higher powers of $a$.) Likewise, the coefficient of $\phi^{8}$ will scale as $\lambda^{4} a^{8-D}$, which means that it is suppressed by $a^{2}$ relative to the coefficient of $\phi^{6}$.

The effective field theory thus consists of three different classes of operators: operators of the form $\phi^{2 n}$, Lorentz-violating operators arising solely from discretization effects, and Lorentzviolating operators due to discretization and quantum effects. These are shown with the scaling of their coefficients in Table 2. At strong coupling, the operators and their scaling remain the same at the scale of the matching of the full theory on to the EFT, although the explicit coefficients are no longer calculable. However, the running of the coefficients down to lower energies is determined by their so-called anomalous dimensions, which depend on the coupling strength. These anomalous dimensions modify the scaling; at weak coupling the modification is small, but at strong coupling it could be larger. (Still, the scaling will remain polynomial.)

From Table 2, one sees that the dominant discretization errors scale as $a^{2}$ in $D=2,3,4$. (In $D=2,3$, errors of type II dominate. In $D=4$, errors of types I and II each scale as $a^{2}$.) These error terms shift scattering probabilities by $\pm \epsilon$ where $\epsilon=O\left(a^{2}\right)$. Limiting $\epsilon$ determines $a$ and $\mathcal{V}=\frac{V}{a^{d}}$, which then determine the overall complexity of the quantum simulation algorithm described in Eq. 1 and Table 1.

In conclusion, we have shown that quantum computers can efficiently calculate scattering probabilities in $\phi^{4}$ theory to arbitrary precision at both weak and strong coupling. Known 


\begin{tabular}{|c|c|c|}
\hline Class & Operators & Scaling of coupling \\
\hline \hline I & $\phi^{2 n}(n \geq 3)$ & $\lambda^{n} a^{2 n-D}$ \\
\hline II & $\phi \partial_{\mathbf{x}}^{2 l} \phi(l \geq 2)$ & $a^{2 l-2}$ \\
\hline III & $\begin{array}{c}\phi^{2 j+1} \partial_{\mathbf{x}}^{2 l} \phi \\
(j \geq 1, l \geq 2)\end{array}$ & $\lambda^{j+1} a^{2 j+2 l+2-D}$ \\
\hline
\end{tabular}

Table 2: Effective field theory operators fall into three classes. The general operator in each class is shown, with the canonical scaling of its coefficient in $D$ spacetime dimensions. Here, $\partial_{\mathbf{x}}^{2 l}=\sum_{i=1}^{d} \partial_{i}^{2 l}$.

classical algorithms take exponential time to do this in the strong-coupling and high-precision regimes. In addition to establishing a new exponential quantum speedup, our algorithm introduces several new techniques. These lead the way towards a quantum algorithm for simulating the Standard Model of particle physics, which has new features, such as chiral fermions and gauge interactions. Such an algorithm would establish that, except for quantum-gravity effects, the standard quantum circuit model suffices to capture completely the computational power of our universe.

\section{References and Notes}

1. R. P. Feynman, International Journal of Theoretical Physics 21, 467 (1982).

2. S. Lloyd, Science 273, 1073 (1996).

3. D. S. Abrams, S. Lloyd, Physical Review Letters 79, 2586 (1997).

4. D. Berry, G. Ahokas, R. Cleve, B. C. Sanders, Communications in Mathematical Physics 270, 359 (2007). ArXiv:quant-ph/0508139.

5. A. Childs, R. Kothari, arXiv:1003.3683 (2010). 
6. C. Zalka, Proceedings of the Royal Society of London A 454, 313 (1998). ArXiv:quant$\mathrm{ph} / 9603026$.

7. S. Wiesner, arXiv:quant-ph/9603028 (1996).

8. T. Byrnes, Y. Yamamoto, Physical Review A 73, 022328 (2006). ArXiv:quant-ph/0510027.

9. M. Lewenstein, et al., Advances in Physics 56, 243 (2007). ArXiv:cond-mat/0606771.

10. M. Johanning, A. Varón, C. Wunderlich, Journal of Physics B 42, 154009 (2009). ArXiv:0905.0118.

11. O. Boada, A. Celi, J. I. Latorre, M. Lewenstein, arXiv:1010.1716 (2010).

12. U. R. Fischer, R. Schützold, Physical Review A 70, 063615 (2004). ArXiv:condmat/0406470.

13. N. C. Menicucci, S. J. Olson, G. J. Milburn, New Journal of Physics 12, 095019 (2010). ArXiv:1005.0434.

14. L. Petersen, Quantum simulations in ion traps - towards simulating the early expanding universe, arXiv:0704.3535 (2006). Diplomathesis.

15. P. D. Nation, M. P. Blencowe, A. J. Rimberg, E. Buks, Physical Review Letters 103, 087004 (2009). ArXiv:quant-ph/0904.2589.

16. J. Casanova, et al., arXiv:1107.5233 (2011).

17. J. Casanova, A. Mezzacapo, L. Lamata, E. Solano, arXiv:1110.3730 (2011).

18. M. Snoek, S. Vandoren, H. T. C. Stoof, Physical Review A 74, 033607 (2006). ArXiv:condmat/0604671. 
19. M. Snoek, M. Haque, S. Vandoren, H. T. C. Stoof, Physical Review Letters 95, 250401 (2005). ArXiv:cond-mat/0505055.

20. J. Glimm, A. Jaffe, Physical Review D 10, 536 (1974).

21. F. Guerra, L. Rosen, B. Simon, Communications in Mathematical Physics 41, 19 (1975).

22. O. A. McBryan, J. Rosen, Communications in Mathematical Physics 51, 97 (1976).

23. K. Osterwalder, R. Sénéor, Helvetica Physica Acta 49, 525 (1976).

24. J.-P. Eckmann, H. Epstein, J. Fröhlich, Annales de l'institut Henri Poincaré (A) Physique théorique 25, 1 (1976).

25. F. Constantinescu, Annals of Physics 108, 37 (1977).

26. M. Suzuki, Physics Letters A 146, 319 (1990).

27. A. Kitaev, W. A. Webb, arXiv:0801.0342 (2008).

28. J. R. Bunch, J. E. Hopcroft, Mathematics of Computation 28, 231 (1974).

29. D. Coppersmith, S. Winograd, Journal of Symbolic Computation 9, 251 (1990).

30. M. E. Peskin, D. V. Schroeder, An Introduction to Quantum Field Theory (Westview, 1995).

31. A. Y. Kitaev, arXiv:quant-ph/9511026 (1995).

32. C. Arzt, Physics Letters B 342, 189 (1995).

33. H. Georgi, Nuclear Physics B 361, 339 (1991).

34. L. Grover, T. Rudolph, arXiv:quant-ph/0208112 (2002).

35. J. C. Le Guillou, J. Zinn-Justin, Phys. Rev. Lett. 39, 95 (1977). 
36. M. Luscher, P. Weisz, Nucl. Phys. B290, 25 (1987).

37. A. Messiah, Quantum Mechanics (Dover, 1999). (Reprint of the two volume edition published by Wiley, 1961-1962.).

38. We thank Alexey Gorshkov for helpful discussions. This work was supported by NSF grant PHY-0803371, DOE grant DE-FG03-92-ER40701, and NSA/ARO grant W911NF-09-10442. Much of this work was done while S.J. was at the Institute for Quantum Information (IQI), Caltech, supported by the Sherman Fairchild Foundation. K.L. was supported in part by NSF grant PHY-0854782. He is grateful for the hospitality of the IQI, Caltech, during parts of this work. 


\section{A Supplementary Material}

\section{A.1 Steps of Algorithm and Comments}

Our quantum algorithm works by the following sequence of steps.

1. Prepare the free vacuum. Improving upon the efficiency of earlier, more general, stateconstruction methods $(6,34)$, Kitaev and Webb developed a quantum algorithm for constructing multivariate Gaussian superpositions (27). For large $\mathcal{V}$, the dominant cost in Kitaev and Webb's method for producing $\mathcal{V}$-dimensional multivariate Gaussians is the computation of the $\mathbf{L D L}^{T}$ decomposition of the inverse covariance matrix, where $\mathbf{L}$ is a unit lower triangular matrix, and $\mathbf{D}$ is a diagonal matrix. This can be done in $\tilde{O}\left(\mathcal{V}^{2.376}\right)$ time with established classical methods $(28,29)$. (The notation $f(n)=\tilde{O}(g(n))$ means $f(n)=O\left(g(n) \log ^{c}(n)\right)$ for some constant $c$.) The computation of the matrix elements of the covariance matrix itself is easy because, for large $V$, the sum

$$
G^{(0)}(\mathbf{x}-\mathbf{y})=\sum_{\mathbf{p} \in \Gamma} L^{-d} \frac{1}{2 \omega(\mathbf{p})} e^{i \mathbf{p} \cdot\left(\mathbf{x}_{i}-\mathbf{x}_{j}\right)}
$$

defining the propagator of the lattice theory is well approximated by an easily evaluated integral.

2. Excite wavepackets. The span of $|\operatorname{vac}(0)\rangle|0\rangle$ and $|\psi\rangle|1\rangle$ is an invariant subspace, on which $H_{\psi}$ acts as

$$
\begin{aligned}
H_{\psi}|\operatorname{vac}(0)\rangle|0\rangle & =|\psi\rangle|1\rangle \\
H_{\psi}|\psi\rangle|1\rangle & =|\operatorname{vac}(0)\rangle|0\rangle
\end{aligned}
$$

Thus,

$$
e^{-i H_{\psi} \pi / 2}|\operatorname{vac}(0)\rangle|0\rangle=-i|\psi\rangle|1\rangle
$$


Hence, by simulating a time evolution according to the Hamiltonian $H_{\psi}$, we obtain the desired wavepacket state $|\psi\rangle$, up to an irrelevant global phase and extra qubit, which can be discarded. After rewriting $H_{\psi}$ in terms of the operators $\phi(\mathbf{x})$ and $\pi(\mathbf{x})$, one sees that simulating $H_{\psi}$ is a very similar task to simulating $H$, and can be done with the same techniques.

The only errors introduced at this step are due to the finite separation distance $\delta$ between wavepackets, and are of order $\epsilon \sim e^{-\delta / m}$. (However, our wavepackets have a constant spread in momentum, and thus differ from the idealization of particles with precisely defined momenta.) The wavepacket preparation thus has complexity scaling linearly with $n_{\text {in }}$, the number of particles being prepared, and necessitates a dependence $V \sim n_{\text {in }} \log (1 / \epsilon)$.

3. Adiabatically turn on the interaction. For $0 \leq s \leq 1$, let

$$
H(s)=\sum_{\mathbf{x} \in \Omega} a^{d}\left[\frac{1}{2} \pi(\mathbf{x})^{2}+\frac{1}{2}\left(\nabla_{a} \phi\right)^{2}(\mathbf{x})+\frac{1}{2} m_{0}^{2}(s) \phi(\mathbf{x})^{2}+\frac{\lambda_{0}(s)}{4 !} \phi(\mathbf{x})^{4}\right]
$$

with $\lambda_{0}(0)=0$. $U_{j}$ is the unitary time evolution induced by $H(t / \tau)$ from $t=\frac{j \tau}{J}$ to $t=\frac{(j+1) \tau}{J}$, namely,

$$
U_{j}=T\left\{\exp \left[-i \int_{j / J}^{(j+1) / J} H(s) \tau d s\right]\right\},
$$

where $T\{\cdot\}$ indicates the time-ordered product. We suppress the dynamical phases by choosing $J$ to be sufficiently large. The choice of a suitable "path" $\lambda_{0}(s), m_{0}^{2}(s)$, and the complexity of this state-preparation process depends in a complicated manner on the parameters in $H(\S \mathrm{A} .5)$.

\section{Simulate Hamiltonian time evolution.}

5. Adiabatically turn off the interaction. The adiabatic turn-off of the coupling is simply the time-reversed version of the adiabatic turn-on. 
6. Measure occupation numbers of momentum modes. For a given $\mathbf{p}$, measurement of $L^{-d} a_{\mathbf{p}}^{\dagger} a_{\mathbf{p}}$ by phase estimation can be implemented with $O\left(\mathcal{V}^{2+\frac{1}{2 k}}\right)$ quantum gates via a $k^{\text {th }}$-order Suzuki-Trotter formula. Furthermore, if we instead simulate localized detectors, the computational cost becomes independent of $V$ (much as the computational cost of creating local wavepackets is independent of $V$ ), but the momentum resolution becomes lower, as dictated by the uncertainty principle.

The allowable rate of adiabatic increase of the coupling constant during state preparation is determined by the physical mass of the theory. In the weakly coupled case, this can be calculated perturbatively. In the strongly coupled case, such a calculation is no longer possible. Thus one is left with the problem of determining how fast one can perform the adiabatic state preparation without introducing errors. Fortunately, one can easily calculate the mass on a quantum computer, as follows. First, one adiabatically prepares the interacting vacuum state at some small $\lambda_{0}$, and measures the energy of the vacuum using phase estimation. The speed at which to increase $\lambda_{0}$ can be chosen perturbatively for this small value of $\lambda_{0}$. Next, one adiabatically prepares the state with a single zero-momentum particle at the same value of $\lambda_{0}$, and measures its energy using phase estimation. Subtracting these values yields the physical mass. This value of the physical mass provides guidance as to the speed of adiabatic increase of the coupling to reach a slightly higher $\lambda_{0}$. Repeating this process for successively higher $\lambda_{0}$ allows one to reach strong coupling, while always having an estimate of mass by which to choose a safe speed for adiabatic state preparation. In addition, mapping out the physical mass as a function of bare parameters (hence, for example, mapping out the phase diagram) may be of independent interest. 


\section{A.2 Efficiency}

To quantify the precision of a simulation, we demand that the probability of a given scattering event in the simulation differ from the true physical probability by no more than $\pm \epsilon$. There are various sources of error: discretization of space, Trotter approximations, imperfect adiabaticity, discretization and cutoff of the field at each site, and imperfect spatial separation of particles in the asymptotic in and out states. In a theory with a non-zero mass, errors due to imperfect particle separation shrink exponentially with distance. Thus, $V$ needs to scale only logarithmically with $\epsilon$. Similarly, by the analysis of $\S$ A.4, the number of qubits per site scales only logarithmically with $\epsilon$. By Eq. 110, the errors resulting from use of a $k^{\text {th }}$-order Suzuki-Trotter formula with $n$ timesteps are $\epsilon \sim n^{-2 k}$. Thus, the complexity scales as $\epsilon^{-1 / 2 k}$. For large $k$, the dominant contributions to scaling with $\epsilon$ are spatial discretization and imperfect adiabaticity.

The effect of spatial discretization is captured by (infinitely many) additional terms in the effective Hamiltonian. Truncation of these terms alters the calculated probability of scattering events. In particular, the two dominant extra terms in the effective Hamiltonian are $\sum_{i} \phi \partial_{i}^{4} \phi$ and $\phi^{6}$ terms, arising from discretization of $\left(\nabla_{a} \phi\right)^{2}$ and quantum effects, respectively. The coefficient of the $\sum_{i} \phi \partial_{i}^{4} \phi$ term is $O\left(a^{2}\right)$, and the coefficient of the $\phi^{6}$ term is $O\left(a^{5-d}\right)$, so that the former dominates for $d=1,2$, whereas the latter makes a comparable contribution for $d=3$. Thus, the overall discretization error is

$$
\epsilon=O\left(a^{2}\right), \quad d=1,2,3 .
$$

(To improve the scaling, one can use better finite differences to approximate the derivative, and/or include the $\phi^{6}$ operator. However, renormalization and mixing of the coefficients make this idea more complicated than it is in standard numerical analysis.)

The diabatic errors at weak coupling are estimated and summarized in $\S$ A.5.1. The errors are quantified by a probability $\epsilon$ of observing stray particles. Substituting the $a \sim \sqrt{\epsilon}$ depen- 
dence from Eq. 17 into Eq. 95 yields $^{4}$

$$
G_{\text {adiabatic }} \sim\left(\frac{1}{\epsilon}\right)^{1+d / 2+o(1)}, \quad d=1,2,3
$$

scaling for the adiabatic state preparation. We use little-o notation to convey precisely that we are neglecting both logarithmic factors and contributions to the exponent that become arbitrarily small as we use higher-order Suzuki-Trotter formulae. The other slow part of the algorithm is the preparation of the free vacuum. This scales as

$$
G_{\text {prep }}=\tilde{O}\left(\mathcal{V}^{2.376}\right)=\tilde{O}\left(a^{-2.376 d}\right)=\tilde{O}\left(\epsilon^{-1.188 d}\right)
$$

where the last equality follows from Eq. 17. Thus, in $d=1$ the adiabatic state preparation is the dominant cost, whereas in $d=2,3$ the preparation of the free vacuum dominates. This leaves a final asymptotic scaling of

$$
G_{\text {total }}=O\left(G_{\text {adiabatic }}+G_{\text {prep }}\right)= \begin{cases}\left(\frac{1}{\epsilon}\right)^{1.5+o(1)}, & d=1, \\ \left(\frac{1}{\epsilon}\right)^{2.376+o(1)}, & d=2, \\ \left(\frac{1}{\epsilon}\right)^{3.564+o(1)}, & d=3 .\end{cases}
$$

The number of quantum gates used to simulate the strongly coupled theory has scaling in $1 /\left(\lambda_{c}-\lambda_{0}\right)$ and $p$ that is dominated by adiabatic state preparation ( $\S$ A.5.2). We also estimate scaling with $n_{\text {out }}$ as follows. For two incoming particles with momenta $\mathbf{p}$ and $-\mathbf{p}$, the maximum number of kinematically allowed outgoing particles is $n_{\text {out }} \sim p$. For continuum behavior, $p=\eta / a$ for constant $\eta \ll 1$. Furthermore, one needs $V \sim n_{\text {out }}$ to obtain good asymptotic out states separated by a distance of at least $\sim 1 / m_{0}$. Thus, $\mathcal{V} \sim n_{\text {out }}^{d+1}$, so one needs $n_{\text {out }}^{2.376(d+1)}$ gates to prepare the free vacuum and, by Eq. 107, $n_{\text {out }}^{2 d+3+o(1)}$ gates to reach the interacting theory adiabatically. (The adiabatic turn-off takes no longer than the adiabatic turn-on.) Hence the total scaling in $n_{\text {out }}$ is dominated by preparation of the free vacuum in three-dimensional spacetime, but by adiabatic turn-on in two-dimensional spacetime. These results are summarized in Table 1.

\footnotetext{
${ }^{4}$ Whether we use Eq. 95 or Eq. 96 affects only the scaling with $V$.
} 


\section{A.3 Mass Renormalization}

The physical, or renormalized, mass as a function of the coupling features prominently in our calculations. For the weak-coupling regime, its form is obtained by perturbation theory. For the strong-coupling regime, we use its known behavior near the phase transition.

At first order in the coupling, the shift of the squared mass is given by $i$ times the one-loop Feynman diagram

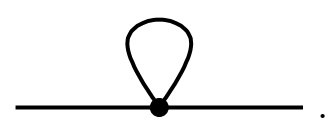

At second order, there is also a contribution from the two-loop diagram

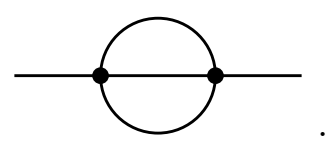

The calculation of these diagrams is quite analogous to standard calculations in perturbative quantum field theory, but there are a couple of differences. First, the propagator is different because of the discretization. Secondly, integrals over components $1, \ldots, d$ (but not component 0 ) of loop momenta are cut off by $\pi / a$, that is, the lattice spacing acts as an ultraviolet regulator. These differences alter the nature the integrals and hence what methods can be used to evaluate them.

The existence of a phase transition in the $\phi^{4}$ theory in $D=2$ or 3 spacetime dimensions was shown rigorously in (20-22). As the system approaches it, thermodynamic functions and correlation functions exhibit power-law behavior, as is characteristic of a second-order phase transition. In particular, for constant $m_{0}^{2}$,

$$
m \sim\left|\lambda_{0}-\lambda_{c}\right|^{\nu},
$$

where $\lambda_{c}$, the critical value of the coupling, depends on $m_{0}^{2}$. 
Empirically, it has been found that systems with second-order phase transitions can be classified into universality classes. Within each class, critical exponents are universal, taking the same values for all systems. (This universality is explained by the concept of the renormalization group.) The $\phi^{4}$ theory is believed to be in the same universality class as the Ising model, for which

$$
\nu= \begin{cases}1, & D=2, \\ 0.63 \ldots, & D=3 .\end{cases}
$$

The value above for $D=3$ has also been obtained directly in the $\phi^{4}$ theory by Borel resummation (35).

In $D=4$ dimensions, in contrast, the believed triviality of the continuum $\phi^{4}$ theory implies that there is no non-trivial fixed point of the renormalization group and hence no phase transition as one varies $\left(m_{0}^{2}, \lambda_{0}\right)$. Moreover, triviality places bounds on the maximum value of the renormalized coupling (36). In particular, strong coupling requires $p a$ to be $O(1)$ : in the continuum-like regime, renormalized perturbation theory should be valid.

\section{A.4 Representation by Qubits}

The required number of qubits per site is

$$
n_{b}=\log \left(1+2\left\lfloor\phi_{\max } / \delta_{\phi}\right\rfloor\right) .
$$

In this section we show that one can simulate processes at energy scale $E$, while maintaining $1-\epsilon$ fidelity to the exact state, with $n_{b}$ logarithmic in $1 / a, 1 / \epsilon$, and $V$. Our analysis is nonperturbative, and thus applies equally to strongly and weakly coupled $\phi^{4}$ theory.

Let $|\psi\rangle$ be the state, expressed in the field representation, namely,

$$
|\psi\rangle=\int_{-\infty}^{\infty} d \phi_{1} \ldots \int_{-\infty}^{\infty} d \phi_{\mathcal{V}} \psi\left(\phi_{1}, \ldots, \phi_{\mathcal{V}}\right)\left|\phi_{1}, \ldots, \phi_{\mathcal{V}}\right\rangle
$$

and let

$$
\left|\psi_{\text {cut }}\right\rangle=\int_{-\phi_{\max }}^{\phi_{\max }} d \phi_{1} \ldots \int_{-\phi_{\max }}^{\phi_{\max }} d \phi_{\mathcal{V}} \psi\left(\phi_{1}, \ldots, \phi_{\mathcal{V}}\right)\left|\phi_{1}, \ldots \phi_{\mathcal{V}}\right\rangle
$$


Then

$$
\left\langle\psi \mid \psi_{\text {cut }}\right\rangle=\int_{-\phi_{\max }}^{\phi_{\max }} d \phi_{1} \ldots \int_{-\phi_{\max }}^{\phi_{\max }} d \phi_{\mathcal{V}} \rho\left(\phi_{1}, \ldots, \phi_{\mathcal{V}}\right),
$$

where $\rho$ is the probability distribution

$$
\rho\left(\phi_{1}, \ldots, \phi_{\mathcal{V}}\right)=\left|\psi\left(\phi_{1}, \ldots, \phi_{\mathcal{V}}\right)\right|^{2}
$$

In other words, $\left\langle\psi \mid \psi_{\text {cut }}\right\rangle=1-p_{\text {out }}$, where $p_{\text {out }}$ is the probability that at least one of $\phi_{1}, \ldots, \phi_{\mathcal{V}}$ is out of the range $\left[-\phi_{\max }, \phi_{\max }\right]$. By the union bound $(\operatorname{Pr}(A \cup B) \leq \operatorname{Pr}(A)+\operatorname{Pr}(B))$,

$$
\left\langle\psi \mid \psi_{\text {cut }}\right\rangle \geq 1-\mathcal{V} \max _{\mathbf{x} \in \Omega} p_{\text {out }}(\mathbf{x}),
$$

where $p_{\text {out }}(\mathrm{x})$ is the probability that $\phi(\mathbf{x})$ is out of the range $\left[-\phi_{\max }, \phi_{\max }\right]$.

Let $\mu_{\phi(\mathbf{x})}$ and $\sigma_{\phi(\mathbf{x})}$ denote the mean and standard deviation of $\phi(\mathbf{x})$ determined by $\rho$. By Chebyshev's inequality, choosing $\phi_{\max }=\mu_{\phi(\mathbf{x})}+c \sigma_{\phi(\mathbf{x})}$ ensures

$$
p_{\text {out }}(\mathbf{x}) \leq \frac{1}{c^{2}} \text {. }
$$

Thus, choosing

$$
\phi_{\max }=O\left(\max _{\mathbf{x} \in \Omega}\left(\mu_{\phi(\mathbf{x})}+\sqrt{\frac{\mathcal{V}}{\epsilon}} \sigma_{\phi(\mathbf{x})}\right)\right)
$$

ensures $\left\langle\psi \mid \psi_{\text {cut }}\right\rangle \geq 1-\epsilon$.

Next, we observe the following, which is straightforward to prove.

Proposition 1 Let $\hat{p}$ and $\hat{q}$ be Hermitian operators on $L^{2}(\mathbb{R})$ obeying the canonical commutation relation $[\hat{p}, \hat{q}]=i \mathbb{1}$. Then the eigenbasis of $\hat{p}$ is the Fourier transform of the eigenbasis of $\hat{q}$.

By Proposition 1, the eigenbasis of $a^{d} \pi(\mathrm{x})$ is the Fourier transform of the eigenbasis of $\phi(\mathbf{x})$. Thus, discretizing $\phi(\mathbf{x})$ in increments of $\delta_{\phi(\mathbf{x})}$ is roughly equivalent to the truncation $-\pi_{\max } \leq \pi(\mathbf{x}) \leq \pi_{\max }$, where

$$
\pi_{\max }=\frac{1}{a^{d} \delta_{\phi(\mathbf{x})}}
$$


By the same argument used to choose $\phi_{\max }$, choosing

$$
\pi_{\max }=O\left(\max _{\mathbf{x} \in \Omega}\left(\mu_{\pi(\mathbf{x})}+\sigma_{\pi(\mathbf{x})} \sqrt{\frac{\mathcal{V}}{\epsilon}}\right)\right)
$$

ensures fidelity $1-\epsilon$ between $|\psi\rangle$ and its truncated and discretized version.

To obtain useful bounds on $\phi_{\max }$ and $\pi_{\max }$, we must bound $\mu_{\phi(\mathbf{x})}, \sigma_{\phi(\mathbf{x})}, \mu_{\pi(\mathbf{x})}$, and $\sigma_{\pi(\mathbf{x})}$. To this end, we make the following straightforward observation.

Proposition 2 Let $M$ be a Hermitian operator and let $|\psi\rangle$ be a quantum state. Then $|\langle\psi|M| \psi\rangle| \leq$ $\sqrt{\left\langle\psi\left|M^{2}\right| \psi\right\rangle}$.

Proof: For brevity, let $\langle Q\rangle=\langle\psi|Q| \psi\rangle$ for any observable $Q$. The operator $(M-\langle M\rangle \mathbb{1})^{2}$ is positive semidefinite. Thus,

$$
\begin{aligned}
0 & \leq\left\langle(M-\langle M\rangle \mathbb{1})^{2}\right\rangle \\
& =\left\langle M^{2}-2\langle M\rangle M+\langle M\rangle^{2} \mathbb{1}\right\rangle \\
& =\left\langle M^{2}\right\rangle-\langle M\rangle^{2} .
\end{aligned}
$$

Applied to the definitions

$$
\begin{aligned}
\mu_{\phi(\mathbf{x})} & =\langle\psi|\phi(\mathbf{x})| \psi\rangle \\
\sigma_{\phi(\mathbf{x})} & =\sqrt{\left\langle\psi\left|\phi(\mathbf{x})^{2}\right| \psi\right\rangle-\langle\psi|\phi(\mathbf{x})| \psi\rangle^{2}} \\
\mu_{\pi(\mathbf{x})} & =\langle\psi|\pi(\mathbf{x})| \psi\rangle \\
\sigma_{\pi(\mathbf{x})} & =\sqrt{\left\langle\psi\left|\pi(\mathbf{x})^{2}\right| \psi\right\rangle-\langle\psi|\pi(\mathbf{x})| \psi\rangle^{2}}
\end{aligned}
$$

Proposition 2 implies that $\mu_{\phi(\mathbf{x})}$ and $\sigma_{\phi(\mathbf{x})}$ are each at most $\sqrt{\left\langle\psi\left|\phi(\mathbf{x})^{2}\right| \psi\right\rangle}$, and $\mu_{\pi(\mathbf{x})}$ and $\sigma_{\pi(\mathbf{x})}$ 
are each at most $\sqrt{\left\langle\psi\left|\pi(\mathbf{x})^{2}\right| \psi\right\rangle}$. Thus, by Eq. 32 and Eq. 34,

$$
\begin{aligned}
& \phi_{\max }=O\left(\max _{\mathbf{x} \in \Omega} \sqrt{\frac{\mathcal{V}}{\epsilon}\left\langle\psi\left|\phi(\mathbf{x})^{2}\right| \psi\right\rangle}\right), \\
& \pi_{\max }=O\left(\max _{\mathbf{x} \in \Omega} \sqrt{\frac{\mathcal{V}}{\epsilon}\left\langle\psi\left|\pi(\mathbf{x})^{2}\right| \psi\right\rangle}\right),
\end{aligned}
$$

so that, by Eq. 25 and Eq. 33,

$$
n_{b}=O\left(\log \left(a^{d} \frac{\mathcal{V}}{\epsilon} \max _{\mathbf{x}, \mathbf{y} \in \Omega} \sqrt{\left\langle\psi\left|\pi(\mathbf{x})^{2}\right| \psi\right\rangle\left\langle\psi\left|\phi(\mathbf{y})^{2}\right| \psi\right\rangle}\right)\right) .
$$

To establish logarithmic scaling of $n_{b}$, we need only prove polynomial upper bounds on $\left\langle\psi\left|\phi(\mathbf{x})^{2}\right| \psi\right\rangle$ and $\left\langle\psi\left|\pi(\mathbf{x})^{2}\right| \psi\right\rangle$. Rather than making a physical estimate of these expectation values, we prove simple upper bounds that are probably quite loose. In the adiabatic state preparation described in $\S$ A.5, the parameters $m_{0}^{2}$ and $\lambda_{0}$ are varied. The following two propositions cover all the combinations of parameters used in the adiabatic preparation and subsequent scattering of both strongly and weakly coupled wavepackets.

Proposition 3 Let $H$ be of the form shown in Eq. 15. Suppose $m_{0}^{2}>0$ and $\lambda_{0} \geq 0$. Let $|\psi\rangle$ be any state of the field such that $\langle\psi|H| \psi\rangle \leq E$. Then $\forall \mathbf{x} \in \Omega$,

$$
\begin{aligned}
\left\langle\psi\left|\phi(\mathbf{x})^{2}\right| \psi\right\rangle & \leq \frac{2 E}{a^{d} m_{0}^{2}} \\
\left\langle\psi\left|\pi(\mathbf{x})^{2}\right| \psi\right\rangle & \leq \frac{2 E}{a^{d}}
\end{aligned}
$$

\section{Proof:}

$$
\begin{aligned}
E & \geq\langle\psi|H| \psi\rangle \\
& =\left\langle\psi\left|\sum_{\mathbf{x} \in \Omega} a^{d}\left[\frac{1}{2} \pi(\mathbf{x})^{2}+\frac{1}{2}\left(\nabla_{a} \phi\right)^{2}(\mathbf{x})+\frac{m_{0}^{2}}{2} \phi(\mathbf{x})^{2}+\frac{\lambda_{0}}{4 !} \phi(\mathbf{x})^{2}\right]\right| \psi\right\rangle \\
& \geq\left\langle\psi\left|a^{d} \frac{m_{0}^{2}}{2} \phi(\mathbf{x})^{2}\right| \psi\right\rangle,
\end{aligned}
$$


where the last inequality follows because all of the operators we have dropped are positive semidefinite. This establishes Eq. 45. Similarly, we can drop all but the $\pi(\mathbf{x})$ term from the right-hand side of Eq. 48, leaving

$$
E \geq\left\langle\psi\left|a^{d} \frac{1}{2} \pi(\mathbf{x})^{2}\right| \psi\right\rangle
$$

which establishes Eq. 46.

Proposition 4 Let $H$ be of the form shown in Eq. 15. Suppose $m_{0}^{2} \leq 0$ and $\lambda_{0}>0$. Let $|\psi\rangle$ be any state of the field such that $\langle\psi|H| \psi\rangle \leq E$. Then $\forall \mathbf{x} \in \Omega$,

$$
\begin{aligned}
\left\langle\psi\left|\phi(\mathbf{x})^{2}\right| \psi\right\rangle & \leq-\frac{24 m_{0}^{2}}{\lambda_{0}}+\sqrt{\frac{36 m_{0}^{4}}{\lambda_{0}^{2}}+\frac{24}{\lambda_{0} a^{d}}\left(E+\frac{3\left(V-a^{d}\right) m_{0}^{4}}{2 \lambda_{0}}\right)} \\
\left\langle\psi\left|\pi(\mathbf{x})^{2}\right| \psi\right\rangle & \leq \frac{2}{a^{d}}\left(E+\frac{3 V m_{0}^{4}}{2 \lambda_{0}}\right)
\end{aligned}
$$

where $V$ is the physical volume.

Proof: The operator

$$
U(\mathbf{x})=\frac{m_{0}^{2}}{2} \phi(\mathbf{x})^{2}+\frac{\lambda_{0}}{4 !} \phi(\mathbf{x})^{4}
$$

is sufficiently simple that we can directly calculate its minimal eigenvalue $U_{\text {min }}$. If $m_{0}^{2} \leq 0$ and $\lambda>0$, then

$$
U_{\min }=-\frac{3 m_{0}^{4}}{2 \lambda_{0}}
$$

Thus, for any state $|\psi\rangle$,

$$
\left\langle\psi\left|\sum_{\mathbf{x} \in \Omega} a^{d} U(\mathbf{x})\right| \psi\right\rangle \geq \frac{-3 V m_{0}^{4}}{2 \lambda_{0}}
$$


Hence, recalling Eq. 15, we obtain

$$
\begin{aligned}
E & \geq\langle\psi|H| \psi\rangle \\
& =\left\langle\psi\left|\sum_{\mathbf{x} \in \Omega} a^{d}\left[\frac{1}{2} \pi(\mathbf{x})^{2}+\frac{1}{2}\left(\nabla_{a} \phi\right)^{2}(\mathbf{x})+\frac{m_{0}^{2}}{2} \phi(\mathbf{x})^{2}+\frac{\lambda_{0}}{4 !} \phi(\mathbf{x})^{4}\right]\right| \psi\right\rangle \\
& \geq\left\langle\psi\left|\sum_{\mathbf{x} \in \Omega} a^{d}\left[\frac{1}{2} \pi(\mathbf{x})^{2}+\frac{1}{2}\left(\nabla_{a} \phi\right)^{2}(\mathbf{x})\right]\right| \psi\right\rangle-\frac{3 V m_{0}^{4}}{2 \lambda_{0}} \\
& \geq\left\langle\psi\left|\frac{a^{d}}{2} \pi(\mathbf{x})^{2}\right| \psi\right\rangle-\frac{3 V m_{0}^{4}}{2 \lambda_{0}}
\end{aligned}
$$

Eq. 58 follows from Eq. 55. Eq. 59 holds (for any choice of $\mathrm{x}$ ) because all of the operators we have dropped are positive semidefinite. This establishes Eq. 52.

Similarly, dropping positive operators from Eq. 57 and using Eq. 55 yield, for any $\mathbf{x}$,

$$
a^{d}\left\langle\psi\left|\left(\frac{m_{0}^{2}}{2} \phi(\mathbf{x})^{2}+\frac{\lambda_{0}}{4 !} \phi(\mathbf{x})^{4}\right)\right| \psi\right\rangle \leq\left(E+\frac{3\left(V-a^{d}\right) m_{0}^{4}}{2 \lambda_{0}}\right) .
$$

Applying Proposition 2 with $M=\phi(\mathbf{x})^{2}$ shows that $\left\langle\psi\left|\phi(\mathbf{x})^{4}\right| \psi\right\rangle \geq\left\langle\psi\left|\phi(\mathbf{x})^{2}\right| \psi\right\rangle^{2}$. Thus,

$$
a^{d}\left[\frac{m_{0}^{2}}{2}\left\langle\psi\left|\phi(\mathbf{x})^{2}\right| \psi\right\rangle+\frac{\lambda_{0}}{4 !}\left\langle\psi\left|\phi(\mathbf{x})^{2}\right| \psi\right\rangle^{2}\right] \leq\left(E+\frac{3\left(V-a^{d}\right) m_{0}^{4}}{2 \lambda_{0}}\right) .
$$

Via the quadratic formula, this implies Eq. 51.

\section{A.5 Adiabatic Preparation of Interacting Wavepackets}

In this section, we analyze the adiabatic state-preparation procedure. To analyze the error due to finite $\tau$ and $J$, we consider the process of preparing a single-particle wavepacket. The procedure performs similarly in preparing wavepackets for multiple particles provided the particles are separated by more than the characteristic length $1 / m$ of the interaction.

The phase induced by $M_{j}$ on the momentum- $p$ eigenstate of $H(s)$ (with energy $E_{p}(s)$ ) is

$$
\theta_{j}(p)=\left(E_{p}\left(\frac{j+1}{J}\right)+E_{p}\left(\frac{j}{J}\right)\right) \frac{\tau}{2 J}-\tau \int_{j / J}^{(j+1) / J} d s E_{p}(s) .
$$


Taylor expanding $E_{p}$ about $s=\left(j+\frac{1}{2}\right) / J$ yields

$$
\theta_{j}(p)=\frac{\tau}{12 J^{3}} \frac{\partial^{2} E_{p}}{\partial s^{2}}+O\left(J^{-5}\right)
$$

Thus the total phase induced is

$$
\begin{aligned}
\theta(p) & =\sum_{j=0}^{J-1} \theta_{j}(p) \\
& \simeq \frac{\tau}{12 J^{2}} \int_{0}^{1} d s \frac{\partial^{2} E_{p}}{\partial s^{2}} \\
& =\left.\frac{\tau}{12 J^{2}} \frac{\partial E_{p}}{\partial s}\right|_{0} ^{1}
\end{aligned}
$$

where the approximation holds for large $J$. For a Lorentz-invariant theory, $E_{p}(s)$ must take the form

$$
E_{p}(s)=\sqrt{p^{2}+m^{2}(s)}
$$

This should be a good approximation for the lattice theory provided the particle momentum satisfies $p \ll 1 / a$. Substituting Eq. 67 into Eq. 66 yields

$$
\left.\theta(p) \simeq \frac{\tau}{24 J^{2}} \frac{\frac{\partial m^{2}}{\partial s}}{\sqrt{p^{2}+m^{2}(s)}}\right|_{0} ^{1}
$$

Next, we consider the effect of this phase shift on a wavepacket centered around momentum $\bar{p}$. If the wavepacket is narrowly concentrated in momentum, then we can Taylor expand $\theta(p)$ to first order about $\bar{p}$ :

$$
\theta(p) \simeq \theta(\bar{p})+\mathcal{D} \cdot(p-\bar{p})
$$

where

$$
\mathcal{D}=\left.\frac{\partial \theta}{\partial p}\right|_{\bar{p}} .
$$

The phase shift $e^{i \mathcal{D} \cdot(p-\bar{p})}$ induces a translation (in position space) of any wavepacket by a distance $\mathcal{D}$. (The second-order term in the Taylor expansion induces broadening.) From Eq. 70 


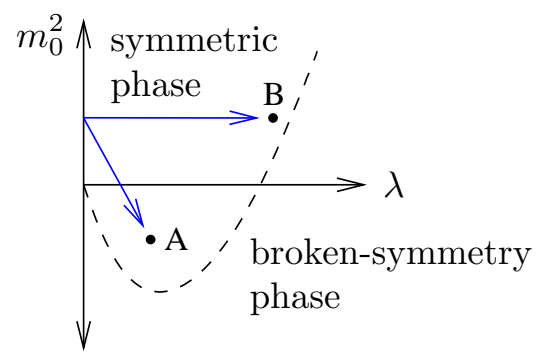

Figure 1: The dashed line illustrates schematically the location of a quantum phase transition of $\phi^{4}$ theory in two and three spacetime dimensions. A and B denote weakly and strongly coupled continuum-like theories, respectively. We prepare them adiabatically by following the arrows starting from the massive free theory $\left(m_{0}^{2}>0, \lambda_{0}=0\right)$. To maintain adiabaticity, the path must not cross the quantum phase transition.

and Eq. 68, we have

$$
\mathcal{D} \simeq\left|\frac{\tau|\bar{p}|}{24 J^{2}} \frac{\frac{\partial m^{2}}{\partial s}}{\left(\bar{p}^{2}+m^{2}(s)\right)^{3 / 2}}\right|_{s=0}^{s=1} \mid .
$$

We next determine the complexity by demanding that the propagation length $\mathcal{D}$ be restricted to some small constant, and that the probability of diabatic particle creation be small. Together, these criteria determine $J$ and $\tau$. We can obtain a tighter bound in the perturbative case than in the general case, so we treat these separately.

\section{A.5.1 Weak Coupling}

In the perturbative continuum limit $a \rightarrow 0, m_{0}^{2}$ is negative. For fixed small $a$, we can adiabatically approach a perturbative continuum-like theory by taking the straight-line path depicted in Fig. 1, namely, the following parameterization of Eq. 15:

$$
\begin{aligned}
m_{0}^{2}(s) & =\left(m^{(1)}\right)^{2}+s \lambda_{0} \mu \\
\lambda_{0}(s) & =s \lambda_{0}
\end{aligned}
$$


Using perturbation theory (see diagram 21), one finds that it is particularly efficient to choose

$$
\mu= \begin{cases}-\frac{1}{8 \pi} \log \left(\frac{64}{m^{2} a^{2}}\right)+\cdots, & d=1, \\ -\frac{r_{0}^{(2)}}{16 \pi^{2}} \frac{1}{a}+\cdots, & d=2, \\ -\frac{r_{0}^{(3)}}{32 \pi^{3}} \frac{1}{a^{2}}+\cdots, & d=3,\end{cases}
$$

so that, at first order in $\lambda_{0}$, the physical mass remains fixed at $m^{(1)}$ for all $s$. Here, $r_{0}^{(2)}=$ $25.379 \ldots$ and $r_{0}^{(3)}=112.948 \ldots$ In the perturbative regime, this should ensure that the path does not cross the quantum phase transition.

To calculate the variation of physical mass with $s$, we must go to second order in $\lambda_{0}$ (see diagram 22). The result is

$$
m^{2}(s)=\left(m^{(1)}\right)^{2}+s^{2} m_{2}^{2}+O\left(\lambda_{0}^{3}\right)
$$

where

$$
m_{2}^{2}= \begin{cases}O\left(\lambda_{0}^{2} /\left(m^{(1)}\right)^{2}\right), & d=1, \\ O\left(\lambda_{0}^{2} \log \left(m^{(1)} a\right)\right), & d=2 \\ O\left(\lambda_{0}^{2} / a^{2}\right), & d=3 .\end{cases}
$$

Substituting Eq. 74 into Eq. 71 yields

$$
\frac{\tau|\bar{p}|}{12 J^{2}} \frac{m_{2}^{2}}{\left(\bar{p}^{2}+\left(m^{(1)}\right)^{2}+m_{2}^{2}\right)^{3 / 2}} \leq \mathcal{D} .
$$

If we are considering a fixed physical process and using successively smaller $a$ to achieve higher precision then, by Eq. 75, it suffices to choose $J$ to scale as

$$
J= \begin{cases}\tilde{O}\left(\sqrt{\frac{m^{(1)} \mathcal{T}}{\lambda_{0} \mathcal{D}}}\right), & d=1 \\ \tilde{O}\left(\sqrt{\frac{\tau}{\lambda_{0} \mathcal{D}}}\right), & d=2 \\ \tilde{O}\left(\sqrt{\frac{a \tau}{\lambda_{0} \mathcal{D}}}\right), & d=3\end{cases}
$$

Note that, for $d=3, J$ is suppressed by $\sqrt{a}$. This is because, as $s$ increases, the (uncancelled) two-loop contribution to the physical mass makes the particle very heavy until $s$ is very close to one. Hence, the particle propagates slowly, and less backward evolution is required. 
To determine $\tau$, we next consider adiabaticity. Let $H(s)$ be any Hamiltonian differentiable with respect to $s$. Let $\left|\phi_{l}(s)\right\rangle$ be an eigenstate $H(s)\left|\phi_{l}(s)\right\rangle=E_{l}(s)$ separated by a non-zero energy gap for all $s$. Let $\left|\psi_{l}(t)\right\rangle$ be the state obtained by Schrödinger time evolution according to $H(t / \tau)$ with initial condition $\left|\psi_{l}(0)\right\rangle=\left|\phi_{l}(0)\right\rangle$. The diabatic transition amplitude to any other eigenstate $H(s)\left|\phi_{k}(s)\right\rangle=E_{k}(s)\left|\phi_{k}(s)\right\rangle(k \neq l)$ is (37)

$$
\left\langle\phi_{k}(s) \mid \psi_{l}(\tau s)\right\rangle \sim \int_{0}^{s} d \sigma \frac{\left\langle\phi_{k}(\sigma)\left|\frac{d H}{d s}\right| \phi_{l}(\sigma)\right\rangle}{E_{l}(\sigma)-E_{k}(\sigma)} e^{i \tau\left(\varphi_{k}(\sigma)-\varphi_{l}(\sigma)\right)}(1+O(1 / \tau)) .
$$

(The integrand is made well-defined by the phase convention $\left\langle\phi_{k}\right| \frac{d\left|\phi_{k}\right\rangle}{d s}=0$.) Here,

$$
\varphi_{l}(s)=\int_{0}^{s} d \sigma E_{l}(\sigma) .
$$

In the case that $E_{l}, E_{k}$, and $\left\langle\phi_{k}\left|\frac{d H}{d s}\right| \phi_{l}\right\rangle$ are $s$-independent, this integral gives

$$
\left\langle\phi_{k}(s) \mid \psi_{l}(\tau s)\right\rangle \sim\left(1-e^{i \tau\left(E_{k}-E_{l}\right) s}\right) \frac{\left\langle\phi_{k}\left|\frac{d H}{d s}\right| \phi_{l}\right\rangle}{-i \tau\left(E_{k}-E_{l}\right)^{2}}\left(1+O\left(1 / \tau^{2}\right)\right) .
$$

In the case that these quantities are approximately $s$-independent, Eq. 80 should hold as an approximation.

In reality, we wish to prepare a wavepacket state, not an eigenstate. However, the wavepacket is well separated from other particles and narrowly concentrated in momentum space. Thus, we shall approximate it as an eigenstate $\left|\phi_{l}(s)\right\rangle$. Furthermore, by our choice of path, the energy gap is kept constant to first order in the coupling, and thus Eq. 80 should be a good approximation to Eq. 78.

Summing the transition amplitudes to some state $\left|\phi_{k}\right\rangle$ from the $J$ steps in in our preparation process, and applying the triangle inequality ${ }^{5}$ yield the following:

$$
\left|\left\langle\phi_{k} \mid \psi_{l}(\tau)\right\rangle\right|=O\left(\frac{1}{\tau} \sum_{j=0}^{J}\left|\frac{\left\langle\phi_{k}(j / J)\left|\frac{d H}{d s}\right| \phi_{l}(j / J)\right\rangle}{\left(E_{k}(j / J)-E_{l}(j / J)\right)^{2}}\right|\right) .
$$

\footnotetext{
${ }^{5}$ The $O(J)$ scaling obtained by the triangle inequality can be confirmed by a more detailed calculation taking into account the relative phases of the contributions to the total transition amplitude.
} 
The $j=0$ term in this sum can be evaluated exactly, because it arises from the free theory. At $j \neq 0$ the theory is no longer exactly solvable. However, one obtains the lowest-order contribution to the matrix element $\left\langle\mathbf{p}_{1}, \mathbf{p}_{2}, \mathbf{p}_{3}, \mathbf{p}_{4} ; s=1\left|\phi^{4}\right| \operatorname{vac}(1)\right\rangle$ in renormalized perturbation theory simply by taking the $j=0$ expression and replacing $m_{0}$ with the physical mass and $\lambda_{0}$ with the physical coupling. Our adiabatic path Eq. 72 is designed so that the physical mass at $s=1$ matches the bare mass at $j=0$ (at least to first order in $\lambda_{0}$ ). Furthermore, the physical coupling differs from the bare coupling only by a logarithmically divergent (in $a$ ) correction for $d=3$ and non-divergent corrections for $d=1,2$. Thus we can make the following approximation:

$$
\left|\left\langle\phi_{k} \mid \psi_{l}(\tau)\right\rangle\right|=\tilde{O}\left(\frac{J}{\tau}\left|\frac{\left\langle\phi_{k}(0)\left|\frac{d H}{d s}\right| \phi_{l}(0)\right\rangle}{\left(E_{k}(0)-E_{l}(0)\right)^{2}}\right|\right) .
$$

Diabatic errors come in two types, creation of particles from the vacuum, and splitting of the incoming particles. The matrix element in the numerator of Eq. 82 can correspondingly be decomposed as the sum of two contributions. We first consider particle creation from the vacuum, approximating $\left|\phi_{j}(s)\right\rangle$ as $|\operatorname{vac}(s)\rangle$.

By Eq. 72,

$$
\frac{d H}{d s}=\sum_{\mathbf{x} \in \Omega} a^{d}\left[\frac{\lambda_{0}}{4 !} \phi^{4}(\mathbf{x})+\lambda_{0} \mu \phi^{2}(\mathbf{x})\right] .
$$

Substituting this into the numerator of Eq. 82, setting $\left|\phi_{l}(0)\right\rangle=|\operatorname{vac}(0)\rangle$, and expanding $\phi$ in terms of creation and annihilation operators show that the only potentially non-zero transition amplitudes are to states $\left|\phi_{k}(0)\right\rangle$ of two or four particles. The transition amplitude to states of four particles arise solely from the $\phi^{4}$ term in $\frac{d H}{d s}$. The transition amplitude to states of two particles has contributions from the $\phi^{4}$ term and the $\phi^{2}$ term in $\frac{d H}{d s}$. These actually cancel, because of our choice of $\mu$. (Note that this requires tuning of $\mu$.) At $s=0$, the numerator of Eq. 82 is therefore the following:

$$
\left\langle\mathbf{p}_{1}, \mathbf{p}_{2}, \mathbf{p}_{3}, \mathbf{p}_{4}\left|\frac{\lambda_{0}}{4 !} \sum_{\mathbf{x} \in \Omega} a^{d} \phi^{4}(\mathbf{x})\right| \operatorname{vac}(0)\right\rangle=\frac{\lambda_{0} \delta_{\mathbf{p}_{1}+\mathbf{p}_{2}+\mathbf{p}_{3}+\mathbf{p}_{4}, 0}}{4 V \sqrt{\omega\left(\mathbf{p}_{1}\right) \omega\left(\mathbf{p}_{2}\right) \omega\left(\mathbf{p}_{3}\right) \omega\left(\mathbf{p}_{4}\right)}}
$$


We obtain the probability of excitation due to creation of four particles from the vacuum by squaring the amplitude estimated above, and then summing over all allowed combinations of the four outgoing momenta:

$$
P_{\text {create }} \sim \sum_{\mathbf{p}_{1}, \mathbf{p}_{2}, \mathbf{p}_{3}, \mathbf{p}_{4} \in \Gamma} \frac{J^{2} \lambda_{0}^{2} \delta_{\mathbf{p}_{1}+\mathbf{p}_{2}+\mathbf{p}_{3}+\mathbf{p}_{4}, 0}}{V^{2} \tau^{2}\left(\omega\left(\mathbf{p}_{1}\right)+\omega\left(\mathbf{p}_{2}\right)+\omega\left(\mathbf{p}_{3}\right)+\omega\left(\mathbf{p}_{4}\right)\right)^{4} \omega\left(\mathbf{p}_{1}\right) \omega\left(\mathbf{p}_{2}\right) \omega\left(\mathbf{p}_{3}\right) \omega\left(\mathbf{p}_{4}\right)} .
$$

This sum is difficult to evaluate exactly; instead, we shall simply estimate its asymptotic scaling. The question is, with which parameter should we consider scaling? There are at least three regimes in which classical methods for computing scattering amplitudes break down or are inefficient: strong coupling, large numbers of external particles, and high precision. In this section we are considering only weak coupling (that is, $\lambda / m^{4-D} \ll 1$ ), leaving discussion of strong coupling until the next section. For an asymptotically large number of external particles, the efficiency of our algorithm depends upon strong coupling, for the following reason. A connected Feynman diagram involving $n$ external particles must have at least $v=O(n)$ vertices, so the amplitude for such a process is suppressed by a factor of $\left(\frac{\lambda}{E^{4-D}}\right)^{v}$, where $E$ is the energy scale of the process. Since $E \geq m$, many-particle scattering events are exponentially rare at weak coupling, and thus cannot be efficiently observed in experiments or simulations. This leaves the high-precision frontier. Recall that the perturbation series used in quantum field theory are asymptotic but not convergent. Thus, perturbative methods cannot be extended to arbitrarily high precision.

Hence, in this section we consider the quantum gate complexity of achieving arbitrarily high precision. To do so, one chooses $a$ small to obtain small discretization errors, $V$ large to obtain better particle separation, $\tau$ long to improve adiabaticity, and $J$ large enough to limit unwanted particle propagation as the interaction is turned on. Thus, we wish to know the scaling of $P_{\text {create }}$ with $a, \tau, V$, and $J$. In this context, we consider $m, \lambda$, and $\left|\mathbf{p}_{1}\right|$ to be constants. 
We now estimate the scaling of $P_{\text {create }}$ as $a \rightarrow 0$.

$$
\begin{aligned}
& P_{\text {create }} \sim \frac{J^{2}}{V^{2} \tau^{2}} \sum_{\mathbf{p}_{1}, \mathbf{p}_{2}, \mathbf{p}_{3} \in \Gamma} \\
& \frac{\lambda_{0}^{2}}{\left(\omega\left(\mathbf{p}_{1}\right)+\omega\left(\mathbf{p}_{2}\right)+\omega\left(\mathbf{p}_{3}\right)+\omega\left(-\mathbf{p}_{1}-\mathbf{p}_{2}-\mathbf{p}_{3}\right)\right)^{4} \omega\left(\mathbf{p}_{1}\right) \omega\left(\mathbf{p}_{2}\right) \omega\left(\mathbf{p}_{3}\right) \omega\left(-\mathbf{p}_{1}-\mathbf{p}_{2}-\mathbf{p}_{3}\right)} \\
& \simeq \frac{3 J^{2}}{V^{2} \tau^{2}} \sum_{\substack{\mathbf{p}_{1}, \mathbf{p}_{2}, \mathbf{p}_{3} \in \Gamma \\
\left|\mathbf{p}_{1}\right|>\left|\mathbf{p}_{2}\right|,\left|\mathbf{p}_{3}\right|}} \\
& \frac{\lambda_{0}^{2}}{\left(\omega\left(\mathbf{p}_{1}\right)+\omega\left(\mathbf{p}_{2}\right)+\omega\left(\mathbf{p}_{3}\right)+\omega\left(-\mathbf{p}_{1}-\mathbf{p}_{2}-\mathbf{p}_{3}\right)\right)^{4} \omega\left(\mathbf{p}_{1}\right) \omega\left(\mathbf{p}_{2}\right) \omega\left(\mathbf{p}_{3}\right) \omega\left(-\mathbf{p}_{1}-\mathbf{p}_{2}-\mathbf{p}_{3}\right)} \\
& \sim \frac{J^{2}}{V^{2} \tau^{2}} \sum_{\substack{\mathbf{p}_{1}, \mathbf{p}_{2}, \mathbf{p}_{3} \in \Gamma \\
\left|\mathbf{p}_{1}\right|>\left|\mathbf{p}_{2}\right|,\left|\mathbf{p}_{3}\right|}} \frac{\lambda_{0}^{2}}{\omega\left(\mathbf{p}_{1}\right)^{6} \omega\left(\mathbf{p}_{2}\right) \omega\left(\mathbf{p}_{3}\right)} \\
& \leq \frac{J^{2}}{V^{2} \tau^{2}} \sum_{\mathbf{p}_{1}, \mathbf{p}_{2}, \mathbf{p}_{3} \in \Gamma} \frac{\lambda_{0}^{2}}{\omega\left(\mathbf{p}_{1}\right)^{6} \omega\left(\mathbf{p}_{2}\right) \omega\left(\mathbf{p}_{3}\right)} \\
& \simeq \frac{V J^{2}}{\tau^{2}} \int_{\Gamma} d^{d} p_{1} \int_{\Gamma} d^{d} p_{2} \int_{\Gamma} d^{d} p_{3} \frac{\lambda_{0}^{2}}{\omega\left(\mathbf{p}_{1}\right)^{6} \omega\left(\mathbf{p}_{2}\right) \omega\left(\mathbf{p}_{3}\right)} \\
& = \begin{cases}\tilde{O}\left(\frac{V J^{2}}{\tau^{2}}\right), & d=1,2, \\
\tilde{O}\left(\frac{V J^{2}}{\tau^{2} a}\right), & d=3 .\end{cases}
\end{aligned}
$$

By Eq. 77 and Eq. 86,

$$
P_{\text {create }}=\tilde{O}\left(\frac{V}{\tau}\right), \quad d=1,2,3 .
$$

Next, we consider the process in which the time dependence of the $\phi^{4}$ term causes a single particle to split into three. For this process, the relevant matrix element is

$$
\left\langle\mathbf{p}_{2}, \mathbf{p}_{3}, \mathbf{p}_{4}\left|\frac{\lambda_{0}}{4 !} \sum_{\mathbf{x} \in \Omega} a^{d} \phi^{4}(\mathbf{x})\right| \mathbf{p}_{1}\right\rangle=\frac{\lambda_{0} \delta_{\mathbf{p}_{2}+\mathbf{p}_{3}+\mathbf{p}_{4}, \mathbf{p}_{1}}}{4 V \sqrt{\omega\left(\mathbf{p}_{1}\right) \omega\left(\mathbf{p}_{2}\right) \omega\left(\mathbf{p}_{3}\right) \omega\left(\mathbf{p}_{4}\right)}},
$$

where $\mathbf{p}_{1}$ is the momentum of the incoming particle. By our choice of path, the physical mass is $s$-independent to first order in the coupling, and the $s$ dependence of the coupling is only logarithmically divergent as $a \rightarrow 0$. Thus, by Eq. 81,

$$
P_{\text {split }} \sim \frac{J^{2}}{\tau^{2} V^{2}} \sum_{\mathbf{p}_{2}, \mathbf{p}_{3}, \mathbf{p}_{4} \in \Gamma} \frac{\lambda_{0}^{2} \delta_{\mathbf{p}_{2}+\mathbf{p}_{3}+\mathbf{p}_{4}, \mathbf{p}_{1}}}{\left(\omega\left(\mathbf{p}_{2}\right)+\omega\left(\mathbf{p}_{3}\right)+\omega\left(\mathbf{p}_{4}\right)-\omega\left(\mathbf{p}_{1}\right)\right)^{4} \omega\left(\mathbf{p}_{1}\right) \omega\left(\mathbf{p}_{2}\right) \omega\left(\mathbf{p}_{3}\right) \omega\left(\mathbf{p}_{4}\right)} .
$$


Let us now examine the divergence structure of $P_{\text {split }}$ as $a \rightarrow 0$. In the limit of large volume, the sum converges to the following integral:

$$
\frac{2 J^{2}}{\tau^{2}} \int_{\Gamma} d^{d} p_{2} \int_{\Gamma} d^{d} p_{3} \frac{\lambda_{0}^{2}}{\left(\omega\left(\mathbf{p}_{2}\right)+\omega\left(\mathbf{p}_{3}\right)+\omega\left(\mathbf{p}_{1}-\mathbf{p}_{2}-\mathbf{p}_{3}\right)-\omega\left(\mathbf{p}_{1}\right)\right)^{4} \omega\left(\mathbf{p}_{1}\right) \omega\left(\mathbf{p}_{2}\right) \omega\left(\mathbf{p}_{3}\right) \omega\left(\mathbf{p}_{1}-\mathbf{p}_{2}-\mathbf{p}_{3}\right)} .
$$

If this were divergent as $a \rightarrow 0$, then by approximating the integrand with its value at large $\left|\mathbf{p}_{2}\right|$ and $\left|\mathbf{p}_{3}\right|$, we would be able to isolate the divergence:

$$
P_{\text {split }} \sim \frac{J^{2} \lambda_{0}^{2}}{\tau^{2} \omega\left(\mathbf{p}_{1}\right)} \int_{\Gamma} d^{d} p_{2} \int_{\Gamma} d^{d} p_{3} \frac{1}{\left(\left|\mathbf{p}_{2}\right|+\left|\mathbf{p}_{3}\right|+\left|\mathbf{p}_{2}+\mathbf{p}_{3}\right|\right)^{4}\left|\mathbf{p}_{2}\right|\left|\mathbf{p}_{3}\right|\left|\mathbf{p}_{2}+\mathbf{p}_{3}\right|}
$$

However, for $d=1,2,3$ this is convergent as $a \rightarrow 0$. Thus, recalling Eq. 77, we obtain

$$
P_{\text {split }}=O\left(\frac{J^{2}}{\tau^{2}}\right)= \begin{cases}\tilde{O}\left(\frac{1}{\tau}\right), & d=1,2 \\ \tilde{O}\left(\frac{a}{\tau}\right), & d=3\end{cases}
$$

We can consider two criteria regarding diabatic particle creation. If our detectors are localized, we may be able to tolerate a low constant density of stray particles created during state preparation. This background is similar to that encountered in experiments, and may not invalidate conclusions from the simulation. Alternatively, one could adopt a strict criterion by demanding that, with high probability, not even one stray particle is created in the volume being simulated during state preparation. This strict criterion can be quantified by demanding that the adiabatically produced state has an inner product of at least $1-\epsilon$ with the exact state. This parameter $\epsilon$ is thus directly comparable with that used in $\S$ A.4, and the two sources of error can be added. Applying the strict criterion, we demand that $P_{\text {split }}$ and $P_{\text {create }}$ each be of order $\epsilon$, and obtain

$$
\tau_{\text {strict }}=\tilde{O}\left(\frac{V}{\epsilon}\right), \quad d=1,2,3 .
$$

Applying the more lenient criterion that $P_{\text {create }} / V$ and $P_{\text {split }}$ each be of order $\epsilon$ yields

$$
\tau_{\text {lenient }}=\tilde{O}\left(\frac{1}{\epsilon}\right), \quad d=1,2,3 .
$$


For a $k^{\text {th }}$-order Suzuki-Trotter formula, the asymptotic scaling of the total number of gates needed for adiabatic state preparation is $\left.O\left((\mathcal{V} \tau)^{1+\frac{1}{2 k}}\right)=O\left(\left(V \tau / a^{d}\right)^{1+\frac{1}{2 k}}\right)\right)$. Thus,

$$
\begin{aligned}
& G_{\text {adiabatic }}^{\text {strict }}=\tilde{O}\left(\left(\frac{V^{2}}{a^{d} \epsilon}\right)^{1+\frac{1}{2 k}}\right), \\
& G_{\text {adiabatic }}^{\text {lenient }}=\tilde{O}\left(\left(\frac{V}{a^{d} \epsilon}\right)^{1+\frac{1}{2 k}}\right) .
\end{aligned}
$$

\section{A.5.2 Strong Coupling}

In two and three spacetime dimensions, we can obtain a strongly coupled (that is, nonperturbative) field theory by approaching the phase transition ( $\S$ A.3). As in the case of weak coupling, the necessary time for adiabatic state preparation depends on various physical parameters of the system being simulated, including the momentum of the incoming particles, the volume, the strength of the final coupling, the number of spatial dimensions, and the physical mass. To keep the discussion concise, we restrict our discussion to the case of ultrarelativistic incoming particles, with coupling strength close to the critical value. Under these conditions, the incoming particles can produce a shower of many $\left(n_{\text {out }} \sim p / m\right)$ outgoing particles. Because of the strong coupling, perturbation theory is inapplicable, and, even if it could be used, would take exponential computation in the number of outgoing particles.

In the strongly coupled case, we vary the Hamiltonian 15 with $s$ by keeping the bare mass constant at $m_{0}$ and setting the bare coupling to $s \lambda_{0}$. We choose $\lambda_{0}$ only slightly below the critical value $\lambda_{c}$, so that at $s=1$ the system closely approaches the phase transition, as illustrated in Fig. 1. Examining Eq. 68 suggests that we can estimate phase errors by understanding the behavior of $m^{2}(s)$ at $s=0$ and $s=1$, without needing to know exactly what happens in between. From Eq. 73,

$$
\left.\frac{d m^{2}}{d s}\right|_{s=0}= \begin{cases}\frac{\lambda_{0}}{8 \pi} \log \left(\frac{64}{m_{0}^{2} a^{2}}\right) & d=1 \\ \frac{25.379}{16 \pi^{2}} \frac{\lambda_{0}}{a} & d=2\end{cases}
$$


and, from Eq. 23 and Eq. 24,

$$
\left.\frac{d m^{2}}{d s}\right|_{s=1} \sim \begin{cases}-2\left(\lambda_{c}-\lambda_{0}\right) & d=1 \\ -1.26\left(\lambda_{c}-\lambda_{0}\right)^{0.26} & d=2 .\end{cases}
$$

Thus, Eq. 71 yields

$$
J=\tilde{O}\left(\sqrt{\frac{\tau \lambda_{0}}{a^{d-1} p^{2} \mathcal{D}}}\right), \quad d=1,2,
$$

under the assumption that $\left(\lambda_{c}-\lambda_{0}\right)$ is very small.

The result 68 rests on two approximations, a Taylor expansion to second order in Eq. 63, and an approximation of a sum by an integral in Eq. 66. The validity conditions for these approximations become most stringent at $s=1$, where the derivatives of $m^{2}$ with respect to $s$ become large. Working out the $O\left(J^{-4}\right)$ term in Eq. 68 at $s=1$, one finds that it will be much smaller than the $O\left(J^{-2}\right)$ term at $s=1$ provided

$$
J \gg \frac{1}{\lambda_{c}-\lambda_{0}} .
$$

Similarly, higher-order terms in the Taylor expansion are suppressed by additional powers of $\frac{1}{J\left(\lambda_{c}-\lambda_{0}\right)}$. The criterion 100 also suffices to justify the approximation of the sum by an integral in Eq. 66.

We must next consider adiabaticity to determine $\tau$. In the ultrarelativistic limit, the relevant energy gap $\gamma$ is $\sim \frac{m^{2}}{p}$. This takes its minimum value at $s=1$, namely,

$$
\gamma_{\text {min }} \simeq \begin{cases}\frac{\left(\lambda_{c}-\lambda_{0}\right)^{2}}{p}, & d=1 \\ \frac{\left(\lambda_{c}-\lambda_{0}\right)^{1.26}}{p}, & d=2 .\end{cases}
$$

Unlike in the perturbative case, we cannot make a detailed quantitative analysis, but under the condition 100, we should again be able to apply the traditional adiabatic criterion and obtain a diabatic transition amplitude scaling as $\frac{J}{\tau \gamma^{2}}$. Thus, to keep the error probability at some small constant $\epsilon$, we have

$$
\tau \sim \frac{J}{\gamma^{2} \sqrt{\epsilon}} .
$$


We now consider asymptotic scaling with $p$ for fixed $\lambda_{0}$. To achieve continuum-like behavior we need $a \ll \frac{1}{p}$. Thus Eq. 99 yields

$$
J \sim \tau^{1 / 2} p^{(d-3) / 2}, \quad d=1,2
$$

Substituting Eq. 100 and Eq. 101 into Eq. 102, we see that we need

$$
\tau \gtrsim p^{2}, \quad d=1,2
$$

Substituting Eq. 103 and Eq. 101 into Eq. 102, we see that we need

$$
\tau \gtrsim p^{d+1}, \quad d=1,2
$$

The scaling $\tau=O\left(p^{d+1}\right)$ for $d=1,2$ suffices to satisfy both conditions 104 and 105 . Thus, by $\S$ A.6, the total number of gates scales as

$$
\begin{aligned}
G_{\text {strong }} & =O\left((V \tau)^{1+o(1)} p^{d+1+o(1)}\right) \\
& =O\left(V^{1+o(1)} p^{2 d+2+o(1)}\right)
\end{aligned}
$$

for $d=1,2$.

Next, we consider asymptotic scaling with $\left(\lambda_{c}-\lambda_{0}\right)$ for fixed $p$. The $J$ scaling as $\sqrt{\tau}$ in Eq. 99 automatically satisfies the condition 100. Thus, we substitute Eq. 99 into Eq. 102, obtaining

$$
\tau \sim \begin{cases}\left(\frac{1}{\lambda_{c}-\lambda_{0}}\right)^{8}, & d=1 \\ \left(\frac{1}{\lambda_{c}-\lambda_{0}}\right)^{5.04}, & d=2 .\end{cases}
$$

Thus, using a $k^{\text {th }}$-order Suzuki-Trotter formula, we obtain

$$
G_{\text {strong }} \sim \begin{cases}\left(\frac{1}{\lambda_{c}-\lambda_{0}}\right)^{8\left(1+\frac{1}{2 k}\right)}, & d=1, \\ \left(\frac{1}{\lambda_{c}-\lambda_{0}}\right)^{5.04\left(1+\frac{1}{2 k}\right)}, & d=2 .\end{cases}
$$

Note that one could improve this scaling by choosing a more optimized adiabatic state-preparation schedule, which slows down as the gap gets smaller. 


\section{A.6 Suzuki-Trotter Formulae for Large Lattices}

It appears that, while scaling with $t$ has been thoroughly studied, little attention has been given to scaling of quantum simulation algorithms with the number of lattice sites $\mathcal{V}$. Using a result of Suzuki and elementary Lie algebra theory, we derive linear scaling provided the Hamiltonian is local.

For any even $k$ and any pair of Hamiltonians $A, B$,

$$
\left(e^{i A \alpha_{1} t / n} e^{i B \beta_{1} t / n} e^{i A \alpha_{2} t / n} e^{i \beta_{2} B t / n} \ldots e^{i A \alpha_{r} t / n}\right)^{n}=e^{i(A+B) t}+O\left(t^{2 k+1} / n^{2 k}\right),
$$

where $r=1+5^{k / 2-1}$ and $\alpha_{1}, \ldots, \alpha_{r}, \beta_{1}, \ldots, \beta_{r-1}$ are specially chosen coefficients such that $\sum_{j=1}^{r} \alpha_{j}=1$ and $\sum_{j=1}^{r-1} \beta_{j}=1$ (26). Thus, using the $k^{\text {th }}$-order Suzuki-Trotter formula (Eq. 110), one can simulate evolution for time $t$ with $O\left(t^{\frac{2 k+1}{2 k}}\right)$ quantum gates (4). To determine the $\mathcal{V}$ scaling, we use the following standard theorem (cf. the Baker-Campbell-Hausdorff formula).

Theorem 1 Let $A$ and $B$ be elements of a Lie algebra defined over any field of characteristic 0. Then $e^{A} e^{B}=e^{C}$, where $C$ is a formal infinite sum of elements of the Lie algebra generated by $A$ and $B$.

$A$ and $B$ generate a Lie algebra by commutation and linear combination. Thus, without requiring any explicit calculation, Theorem 1 together with Eq. 110 implies

$$
\left(e^{i A \delta_{1} t / n} e^{i B \delta_{2} t / n} \ldots e^{i A \delta_{r} t / n}\right)^{n}=e^{i(A+B) t}+\Delta_{2 k+1} t^{2 k+1} / n^{2 k}+O\left(n^{-(2 k+1)}\right),
$$

where $\Delta_{2 k+1}$ is a linear combination of nested commutators. In general, $\left\|\Delta_{2 k+1}\right\|$ could be as large as $(\max \{\|A\|,\|B\|\})^{2 k+1}$. However, by the canonical commutation relations, one sees that, for the pair of local Hamiltonians $H_{\phi}, H_{\pi},\left\|\Delta_{2 k+1}\right\|=O(\mathcal{V})$, for any fixed $k$. Thus, one needs only $n=O\left(t^{\frac{2 k+1}{2 k}} \mathcal{V}^{\frac{1}{2 k}}\right)$. Recalling the $O(\mathcal{V})$ cost for simulating each $e^{i H_{\phi} \delta t}$ or $e^{i H_{\pi} \delta t}$, 
one sees that the total number of gates scales as $O\left((t \mathcal{V})^{1+\frac{1}{2 k}}\right)$. Note that this conclusion may be of general interest, as it applies to any lattice Hamiltonian for which non-neighboring terms commute.

In the case of strong coupling, we care not only about how the number of gates scales with $\mathcal{V}$, but also about scaling with $p$. In the presence of high-energy incoming particles, the field can have large distortions from its vacuum state. For example, if $\langle\psi|\phi(\mathbf{x})| \psi\rangle$ is large, then local terms in $\Delta_{2 k+1}|\psi\rangle$ such as $\pi(\mathbf{x}) \phi(\mathbf{x})^{3}|\psi\rangle$ can become large. We can obtain a heuristic upper bound on this effect by noting that, in the strongly coupled case, $m_{0}^{2}>0$, so each local term in $H$ is a positive operator. Thus, if $\langle\psi|H| \psi\rangle \leq E$, then the expectation value of each of the local terms is bounded above by $E$. Using $E$ as a simple estimate of the maximum magnitude of a local term, we see that $\Delta_{2 k+1}|\psi\rangle$, which is a sum of $O(\mathcal{V})$ terms, each of which is of degree $2 k+1$ in the local terms of $H$, has magnitude at most $O\left(\mathcal{V} E^{2 k+1}\right)$, or in other words $O\left(\mathcal{V} p^{2 k+1}\right)$. Recalling that $a$ scales as a small multiple of $1 / p$, we see that $\Delta_{2 k+1}|\psi\rangle=O\left(V p^{2 k+1+d}\right)$. Thus, $n=O\left(p^{1+(1+d) / 2 k} t^{1+1 / 2 k}\right)$. Each timestep requires $O(\mathcal{V})=O\left(V p^{d}\right)$ gates to implement. Thus, the overall scaling is $O\left(p^{d+1+o(1)}(t V)^{1+o(1)}\right)$ quantum gates to simulate the strongly coupled theory at large $p$. 